\title{
Enquête
}

Archives de la revue Enquête

5 | 1989

Biographie et cycle de vie

\section{Espaces transitionnels de socialisation}

Jean-Louis Legrand

\section{(2) OpenEdition \\ Journals}

Édition électronique

URL : http://journals.openedition.org/enquete/104

DOI : 10.4000/enquete. 104

ISSN : 1953-809X

Éditeur :

Cercom, Éditions Parenthèses

Édition imprimée

Date de publication : 2 mars 1989

\section{Référence électronique}

Jean-Louis Legrand, «Espaces transitionnels de socialisation », Enquête [En ligne], 5 | 1989, mis en ligne le 27 juin 2013, consulté le 19 avril 2019. URL : http://journals.openedition.org/enquete/104 ; DOI : 10.4000/enquete. 104

Ce document a été généré automatiquement le 19 avril 2019. 


\title{
Espaces transitionnels de socialisation
}

\author{
Jean-Louis Legrand
}

1 Traditionnellement la socialisation a été essentiellement envisagée sous le seul angle de l'éducation des enfants. Un manuel de sociologie (Mendras, 1975) s'attache ainsi surtout à «l'étude des méthodes d'élevage, d'éducation et d'enseignement ». Sans nier l'importance de cette socialisation des tout premiers âges de la vie, importance attestée par les découvertes de la psychanalyse, il convient de prendre sérieusement en compte les socialisations de la vie dite « adulte ». Avec P. Berger et T. Luckmann nous distinguerons donc les socialisations primaires, celles de l'enfance par lesquelles on devient membre d'une société, et les secondaires qui permettent à un individu de s'adapter à de nouveaux rôles sociaux.

2 La première conception se fixe au terme de l'adolescence, là où la personne est censée devenir adulte et reproduire elle-même le processus vis-à-vis d'une nouvelle génération, celle des enfants. Bien sûr cette conception s'appuie sur une philosophie de l'éducation vue comme préparation à l'« entrée dans la vie », et avec G. Lapassade (1963) nous avons envisagé comment cette figure de l'adulte-étalon, modèle de maturité et de responsabilité, s'avère un masque trompeur venant écarter celle d'un homme fondamentalement en état d'inachèvement.

De manière rapide l'évidence première d'un schéma des cycles de vie avec ses repères biologiques d'âges et ses repères sociaux semble largement remise en cause. Ces repères ont de plus en plus vite tendance à devenir caduques, que ce soient ceux liés aux situations familiales (mariage, vie maritale) ceux correspondants aux cursus professionnels (apparition/disparition de métiers, de savoirs stables, chômage intermittent) ou encore ceux relatifs aux positions sociales liées à l'âge (post-adolescence et précarité sociale, préretraite, $4 \mathrm{e}$ âge...).

De façon diffuse ce thème de la mutation accélérée des rôles sociaux est dans l'« air du temps» et cela s'accompagne d'un bouleversement des valeurs correspondantes. Certaines valeurs transmises par des générations parentales peuvent vite s'avérer dépassées, inadéquates, inopérantes pour faire face à des situations nouvelles. Dès lors il 
n'est guère étonnant que celles mises au goût du jour privilégient la flexibilité, l'adaptabilité, la souplesse, etc.

5 Dans l'histoire contemporaine, une des manifestations les plus criantes de ce phénomène a été la contestation de la fin des années 1960 qui remettait en cause l'autorité «naturelle » de l'adulte responsable. De manière plus discrète mais tout aussi importante la notion d'éducation permanente est passée dans les mœurs et dans les textes officiels (la loi inspirée par J. Delors imposant le $1 \%$ patronal date de 1971). Du côté des rapports hommes/femmes, des bouleversements importants interviennent là aussi.

Ces constatations relèvent bien entendu d'un haut niveau de généralité. Mais dès lors que l'on se trouve aux prises avec une inadéquation entre les valeurs reçues en héritage et celles requises pour s'adapter à des rôles sociaux incertains, cette question des socialisations secondaires prend toute son importance. Suivant Max Weber pour qui l'identité et la socialisation sont, sous un angle sociologique, des notions relatives et flottantes, «il existe en réalité toute une gamme de transitions continues entre les deux termes de l'alternative apparemment exclusive du point de vue logique, celui de la persistance d'une socialisation (Vergesellschaftung) et celui de sa disparition» (1965, p. 353).

7 Le sociologue se doit aussi de s'interroger, de manière très concrète, sur les lieux et les temps de ces socialisations en mouvement. Où et quand se gèrent ces transitions ? En quoi les modifications sont dans la logique de l'ancienne structure sociale ou dans celle de l'apparition d'une nouvelle? Si l'on poursuit la ligne de pensée de Durkheim à propos de l'anomie (dissolution des normes sociales, difficultés d'intégration), on peut penser qu'il y a, pour faire face au changement, un travail à la fois psychologique et social qui s'opère dans des lieux spécifiques. Autrement dit des espaces transitionnels de socialisation. Nous entendons par là des groupes restreints ou plus larges, qui de manière transitoire favorisent une socialisation de leurs membres, quand bien même le but du groupe est ailleurs.

8 Certes il y a bien des lieux qui directement se donnent un tel objectif: je pense aux séminaires de formation permanente, aux multiples stages d'insertion ou d'orientation, aux contrats temporaires et précaires permettant l'inscription dans une entreprise, une collectivité professionnelle, etc. Mais, souvent de manière paradoxale, il existe des lieux ayant une fonction socialisatrice non affichée, dont le but, l'activité, l'orientation sont ailleurs: groupe de loisirs (musique, théâtre, bandes), groupes d'initiation technique (micro-informatique), groupes thérapeutiques, groupes religieux; sans parler des tribus familiales ou néofamiliales, des réseaux affectifs d'amitié qui ont un caractère moins éphémère.

9 J'insiste ici sur le paradoxe qui traverse ce processus. Ainsi, reprenant les travaux d'autres sociologues des religions, Jean Séguy montre bien comment peut s'opérer, dans des institutions aussi apparemment marginales que des sectes, une socialisation utopique non seulement à des valeurs qui leur sont propres mais aussi aux valeurs dominantes dans la société. Dans le registre d'une sociologie générale, Michel Maffesoli insiste, quant à lui, sur l'importance d'une ambiance tribale contemporaine, là où la logique de l'identité (professionnelle, politique) a tendance à se dissoudre mais où prévalent des identifications transitoires correspondant à une théâtralité de la vie sociale. 
De manière à ancrer mon propos, je présenterai désormais certains résultats d'une longue étude empirique qui a donné lieu à une thèse d'État. Cette étude porte sur une communauté d'une douzaine de jeunes "adultes " ayant expérimenté des thérapies à médiation corporelle et émotionnelle : bio-énergie, Gestalt, thérapie par le cri, rebirthing... Cette expérience communautaire située en 1978-1979 est historiquement à la charnière d'un mouvement communautaire finissant et en pleine ascension d'une nouvelle culture psychologique (cf. Castel \& Le Cerf, 1980) mettant en avant le corps, le travail sur soi. L'approche méthodologique que j'ai suivie est triple : a) tout d'abord le suivi longitudinal par l'établissement des trajectoires biographiques avant la période communautaire et après, soit six ans plus tard ; $b$ ) ensuite il y a l'histoire de vie du groupe communautaire, autrement dit l'étude de la période des deux ans de vie commune; $c$ ) puis de manière hétérogène aux deux approches précédentes, intervient une contextualisation sociohistorique de cette expérience avec, en amont le mouvement contre-culturel des communautés, et en aval le phénomène social représenté par les pratiques thérapeutiques psycho-corporelles. Cette tri-dimensionnalité méthodologique permet de lire l'histoire du groupe au travers des biographies mais surtout d'analyser sa fonction socialisatrice d'un point d'observation situé six ans plus tard, le tout daté et placé dans le contexte historique et social de mouvements sociaux déterminés.

11 Tout d'abord voyons qui sont ces communautaires, quelles sont leurs caractéristiques sociales. Sur dix-huit personnes ayant séjourné un certain temps à la communauté, la fourchette des âges est de 22 à 32 ans, avec une moyenne se situant entre 26 et 27 . Nous avons donc affaire à des jeunes adultes qui ne sont plus, pour la grande majorité, étudiants, qui ont tous une expérience du monde du travail, de la vie en couple pour la plupart, du mariage pour certains. Seulement quatre d'entre eux ont des enfants (2 à 4 ans). Les sensibilités idéologiques proviennent, au début des années 1970, d'un militantisme d'extrême gauche, qui par la suite s'essouffle et est partiellement relayé par la préoccupation écologique et l'intérêt pour le Soi (Barbier, 1981). Les perspectives de libération personnelle, psychologique et sexuelle trouvent à se concrétiser dans la pratique des groupes d'expression émotionnelle importés des USA.

12 Si l'on regarde les origines sociales des communautaires, on s'aperçoit que la plupart des familles n'appartiennent pas aux catégories ouvrières ou employés. Nombreux sont les artisans-commerçants-petits entrepreneurs, ou encore les catégories supérieures: industriels, professions libérales. On remarque que la plupart des parents ont un rapport à l'initiative dans le travail et à la reconnaissance sociale qui est radicalement différent des salariés du tertiaire, catégorie dans laquelle se retrouvent en règle générale les communautaires. Il y a une cassure entre d'une part les espoirs véhiculés par la génération parentale quant à la portée des études et d'autre part les situations effectives qui ne suscitent pas l'enthousiasme (Lacroix, 1981). La production de valeurs antiautoritaires et utopiques sont le signe d'un tel décalage; les désertions de leurs rôles sociaux, la propension à ne pas vouloir être «rangé », seraient fonction d'un héritage impossible qui perpétuerait, par d'autres voies que l'insertion professionnelle, une éthique de l'initiative : « être son propre patron ».

13 Dans la période précédant juste l'entrée en communauté, on peut parler d'une forte désaffiliation professionnelle (petits boulots, chômage, situations marginales). Cette période fait, pour beaucoup, suite à une rupture de fraîche date dans une relation de couple. La conjugaison de ces éléments va dans le sens d'une crise d'identité très forte, versant psychologique d'une situation sociale anomique. Pour ces personnes, qui sont 
d'une certaine manière des mutants, l'anomie n'est pas qu'une situation de vacance sociale mais correspond à une intense activité de recherche de soi, de recherche du sens de l'existence. Là où un questionnement ontologique, quasi religieux, est fortement lié à une trajectoire sociale en porte-à-faux.

L'entrée dans une communauté expérimentant ces thérapies constitue indirectement une sorte de défi utopique où de pari existentiel. Et ce d'autant plus que la communauté se veut un espace de dépassement de soi, de liberté sexuelle, où il n'y a pas, au départ, de lieu individuel. La période communautaire peut être structurée en deux périodes successives. Une première où s'opère une plongée dans un tourbillon de pratiques thérapeutiques, de tentatives de sexualités plurielles, de recherches extatiques. Cette période d'éruptive socialité communautaire correspond à une intense recherche personnelle, à une aspiration à une transformation intérieure. À titre de repère significatif, durant cette première période, seules trois personnes sur dix-huit ont occupé une activité salariée à temps plein.

Une deuxième période est dominée par un désir d'individualisation et de désinvestissement de la dynamique groupale ; les thérapies collectives sont abandonnées au profit de thérapies individuelles; la répartition de l'espace attribue à chaque communautaire un endroit à lui. Face aux dangers suscités par la violence mimétique de la jalousie, les rapports affectifs sont de plus en plus stables et codés. Le même phénomène s'observe à propos de l'usage institué des pouvoirs. De plus en plus de personnes passent la porte pour aller trouver un travail qui leur correspond. Au fur et à mesure, la communauté se vide de ses membres qui désirent vivre seuls, jusqu'à ce que la décision d'auto-dissolution soit prise.

16 Si maintenant l'on regarde les trajectoires biographiques durant les six ans qui ont suivi, il apparait que des choix tout à fait déterminants engageant à long terme les sujets prennent leurs origines juste à la fin de cette expérience communautaire. Cela peut être le fait d'avoir des enfants, de fonder une famille, de s'engager dans un lien durable de couple, de se marier, d'effectuer une thérapie de longue haleine, d'entrer dans une voie spirituelle. Cela peut être aussi une réorientation radicale de l'activité sociale dans une nouvelle direction professionnelle ou le fait de commencer un nouveau cycle d'études ou de formation, la plupart du temps en direction des métiers de la relation. À ce propos, il est tout à fait frappant de voir comment ces ex-communautaires sont des amateurs privilégiés de formation permanente ou universitaire et ont un goût prononcé pour le changement. Ce qui est encore caractéristique, ce sont les mutations importantes des valeurs, des systèmes de valeur, des visions du monde.

17 Si l'on s'en tenait à une conception sommaire et clivée opposant irréductiblement norme sociale rigide et « marginalité alternative », il y aurait bien « faillite de l'utopie », « retour à des valeurs traditionnelles ». Pourtant de manière plus subtile, force est de constater qu'il y a mixage entre d'un côté des valeurs et des pratiques issues de cette vague contreculturelle et d'autres plus couramment admises dans la société. Cette société, elle-même, change en direction d'un attrait pour la psychologie, le Soi, promouvant ainsi une nouvelle culture psychologique. Il y a là une mutation socioculturelle essentielle dont témoignent, par exemple, les transformations du rapport au corps, l'emploi du terme «thérapie " par des personnes voulant «être mieux dans leur peau» ou encore l'émergence multidimensionnelle de nouvelles formes néoreligieuses. Ceci est significatif d'une modernité où d'un côté « changer » devient une "nécessité » professionnelle et 
sociale et où, de l'autre côté, ce changement est frileux et nécessite un ancrage dans des «valeurs sûres ».

Dans ce travail sur soi de transformation idéologique (B. M. Berger, 1981), la communauté joue un rôle d'espace transitionnel de socialisation, renforcé par la dimension thérapeutique. La technique du rebirthing représente ici une figure ultime du «travail sur soi ", le changement étant poussé à son extrême puisqu'il postule à un revécu de naissance, à une mort/renaissance symbolique où il est question de «mourir à ceci pour renaître à cela »; l'épreuve corporelle présentant la forme d'un voyage psychédélique par la respiration.

La vie communautaire fait office de plaque tournante qui vient restructurer les parcours existentiels, une sorte d'atelier initiatique sauvage invitant à la reliance (Bolle de Bal, 1984). Suivant la conceptualisation psychanalytique de Winnicot (1975) sur les objets transitionnels, la communauté est ici symboliquement un espace privilégié de jeu, passage entre une phase fusionnelle groupale plongeant l'individu dans une sorte de chaos et une phase d'individuation, de différenciation.

La question du dehors et du dedans, représentée par le franchissement de la porte, met en scène d'un côté une recherche vers l'intérieur de soi, une régression instituée, une manière d'" être en jachère » (Kahn, 1977) et de l'autre une propension à aller dehors, un investissement en direction d'une situation reconnue dans la société, une volonté d'y trouver une place. Au-delà d'une descente aux enfers des conflits psychologiques profonds, une des fonctions des groupes de thérapie est bien de créer un espace théâtral de socialisation. Il s'agit bien là d'une mise en scène, d'une théâtralisation. Une des surprises n'est-elle pas de constater comme une institution aussi apparemment marginale socialise, entre autres à des valeurs dominantes, des personnes identifiées comme marginalisées? Le groupe devient le lieu d'un "chaos socialisateur» qui permet de "refaire surface », structure intermédiaire entre une famille d'origine dont les valeurs se révèlent par trop non opérationnelles et une société incapable de proposer à ses membres une place qui ait un sens pour ceux qui l'occupent.

\section{BIBLIOGRAPHIE}

BARBIER, R. - «Soi comme projet ou la métamorphose militante », in Les révolutions minuscules, Paris, Autrement, 1981, p. 100-110 (« Autrement. Série Mutations » 29).

BERGER, B. M. - The survival of a counterculture. Ideological work and everyday life among rural communard, Berkeley, University of California Press, 1981.

BERGER, P. L., LuCKMANN, T. - La construction sociale de la réalité, Paris, Méridiens/Klincksieck, 1986 (1 ${ }^{\text {re }}$ éd. 1967).

Bolle DE BAL, M. - « Les pages de l'âge. Âge, personne et société », 26 p. multigr. (Exposé introductif au colloque Âges et passages. Les transitions dans la vie, Université libre de Bruxelles, 1984.) 
CASTEL, R., LE CERF, J.-F. - « Le phénomène “psy” et la société française, I. Vers une nouvelle culture psychologique. II. La société de relation. III. L'après-psychanalyse », Le Débat, 1, 2 et 3, 1980.

Durkheim, E. - Le suicide. Étude de sociologie (1897), Paris, Presses universitaires de France, rééd. 1981. - Éducation et sociologie (1922), Paris, PUF, rééd. 1989.

KAHN, M. - « Être en jachère », L'Arc, 69, 1977.

LACRoIX, B. - L'utopie communautaire. Histoire sociale d'une révolte, Paris, PUF, 1981.

LAPASSADE, G. - L'entrée dans la vie. Essai sur l'inachèvement de l'homme, Paris, Minuit, 1963.

LE GRAND, J.-L. - Étude d'une expérience communautaire à orientation thérapeutique. Histoire de vie de groupe. Perspectives sociologiques, Paris, Université Paris-VIII, 1987, thèse de doctorat d'État.

MAFFESOLI, M. - Le temps des tribus. Le déclin de l'individualisme dans les sociétés de masse, Paris, Méridien/Klincksieck, 1988.

MENDRAS, H. - Éléments de sociologie, Paris, Armand Colin, 1975, p. 29.

Moscovici, S. - La machine à faire des dieux. Sociologie et psychologie, Paris, Fayard, 1988.

Pineau, G., PineAu, M.-M. - Produire sa vie. Autoformation et autobiographie, Paris, Edilig, 1983.

SÉGUY, J. - « Les sectes comme mode d'insertion sociale », in Centre de sociologie du protestantisme, Églises et groupes religieux dans la société française. Intégration ou marginalisation, Strasbourg, CERDIC-publications, 1977, p. 293-316. - « La socialisation utopique aux valeurs », Archives de Sciences sociales des Religions, 1980, 50 (1), p. 7-21.

WEBER, M. - « Essai sur quelques catégories de la sociologie comprehensive » (1913), in Essais sur la théorie de la science, Paris, Plon, 1965, p. 327-398.

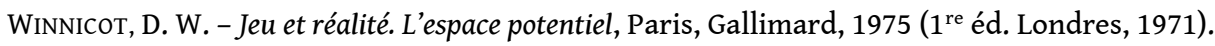

